TITLE:

\title{
Two-photon above-threshold- ionization cross sections of rare-gas atoms by xuv photons
}

$\operatorname{AUTHOR}(S)$ :

Nakajima, T; Watanabe, S

\section{CITATION:}

Nakajima, T ...[et al]. Two-photon above-threshold-ionization cross sections of rare-gas atoms by xuv photons. PHYSICAL REVIEW A 2004, 70(4): 043412.

ISSUE DATE:

2004-10

URL:

http://hdl.handle.net/2433/50417

RIGHT:

Copyright 2004 American Physical Society 
PHYSICAL REVIEW A 70, 043412 (2004)

\title{
Two-photon above-threshold-ionization cross sections of rare-gas atoms by xuv photons
}

\author{
Takashi Nakajima ${ }^{1,2, *}$ and Shuntaro Watanabe ${ }^{2}$ \\ ${ }^{1}$ Institute of Advanced Energy, Kyoto University, Gokasho, Uji, Kyoto 611-0011, Japan \\ ${ }^{2}$ Institute for Solid State Physics (ISSP), The University of Tokyo 5-1-5 Kashiwanoha, Kashiwa, Chiba 277-8581, Japan
}

(Received 1 July 2004; published 21 October 2004)

\begin{abstract}
We have calculated the two-photon above-threshold-ionization cross sections of rare-gas atoms by extreme ultraviolet (xuv) photons in the extended framework of multichannel quantum defect theory. Since free-free dipole moments do not converge in the length gauge, we have made the combined use of the length gauge for small $r$ and the acceleration gauge for large $r$. The two-photon above-threshold ionization cross sections for Xe and Ar associated with the $p^{5}\left[{ }^{2} P_{3 / 2}\right]$ and $p^{5}\left[{ }^{2} P_{1 / 2}\right]$ ionic core states are calculated to be $1.0 \times 10^{-51} \mathrm{~cm}^{4} \mathrm{~s}$ and $2.0 \times 10^{-51} \mathrm{~cm}^{4} \mathrm{~s}$, respectively, for $25-\mathrm{eV}$ photons.
\end{abstract}

DOI: 10.1103/PhysRevA.70.043412

PACS number(s): 42.50.Hz, 42.65.Ky, 32.80.Rm

Above-threshold ionization (ATI) is the successive absorption of an additional number of photons more than the minimum required to ionize an atom [1]. For rare-gas atoms, ATI by infrared-visible photons has been very well studied both experimentally and theoretically. For the theoretical description of the ultrafast laser-atom interactions for rare-gas atoms at small (visible to near-infrared) photon energies, time-dependent calculations with single-active electron (SAE) [2,3] has been found to give good agreements with experimental results. This means that the detailed structure of the atom originated from the fine structure is not very important, in particular in the tunneling regime. Furthermore, the fine structure in the photoelectron spectra is often smeared out because of the large ponderomotive shifts under the intense laser radiation. Among all rare-gas atoms, He is rather special, and as demonstrated in Ref. [4], more rigorous treatment with two active electrons is possible, which again justified the use of SAE for small photon energies.

Due to the recent technological progress in high-order harmonic generation and free-electron lasers, a bright light source is becoming available in the extreme ultraviolet (xuv) wavelength region through high harmonic generation [5-7] or free-electron lasers [8-10]. Provided with such progress, the time is matured to investigate, theoretically as well as experimentally, ATI in the xuv regime [7]. However, one of the potential problems to experimentally observe ATI in the xuv range might be similar to that for alkali atoms [11]: With xuv ( $>24.5 \mathrm{eV}$ which is an ionization energy of He) photons, a single photon is sufficient to ionize any neutral atoms, and the lowest order ATI requires just two photons. This means that the observation of ATI in the xuv range would be a competition between single-photon ionization and twophoton ATI. Typically the single-photon ionization cross section from the ground state is of the order of $10^{-18}-10^{-19} \mathrm{~cm}^{2}$ for any neutral atoms as long as there is no autoionizing resonance nearby. Assuming that the photon energy is $\sim 25 \mathrm{eV}$, the width for single-photon ionization is $\sim 0.25 I\left(\mathrm{sec}^{-1}\right)$ where $I$ is the intensity given in $\mathrm{W} / \mathrm{cm}^{2}$. At present the feasible intensity for xuv photons is of the order

*Email address: t-nakajima@iae.kyoto-u.ac.jp of $10^{12}-10^{13} \mathrm{~W} / \mathrm{cm}^{2}[6,7]$. These facts lead to the conclusion that, in order to observe two-photon ATI the pulse duration must be in the femtosecond time scale so that the depletion of the ground-state atom due to single-photon ionization is not significant.

Apart from the technical difficulty to observe ATI of raregas atoms in the xuv range, there are a few things we should keep in mind: Laser-atom interactions in the xuv range is usually in the multiphoton regime and the ponderomotive shifts are two orders of magnitude smaller than that for the near-infrared-visible photon, indicating that the photoelectron spectra for xuv photons can be much cleaner than those for small photons. Therefore the fine structure of the ionic ground configuration, $p^{5}\left[{ }^{2} P_{3 / 2}\right]$ and $p^{5}\left[{ }^{2} P_{1 / 2}\right]$, can be experimentally resolved [7]. Thus theoretical calculations for the laser-atom interactions of rare-gas atoms beyond the SAE model is desired in the xuv range for the detailed comparison between theory and experiment.

For rare-gas atoms other than $\mathrm{He}$, however, all excited bound states have configurations of $p^{5} n l$ with $n$ and $l$ being the principal quantum number and orbital angular momentum of the excited electron, and continuum states have configurations such as $p^{5} \epsilon l$ and $p^{4} n l \epsilon l^{\prime}$, etc. Due to the several electrons in the open subshells it is practically impossible to take into account all interactions between electrons. This is particularly true for heavier rare-gas atoms where spin-orbit interactions are strong. In the famous classic paper by Kennedy and Manson [12], total as well as partial onephoton ionization cross sections have been calculated using single-particle Hartree-Fock wave functions without spinorbit interactions taken into account. For our purpose such a treatment is insufficient since the inclusion of spin-orbit interaction is crucial.

To tackle such difficulty, an approach we have taken in this paper is an extension of the multichannel quantum defect theory (MQDT) [13]. The advantage of the use of MQDT is that it automatically incorporates not only the spin-orbit interactions but also configuration mixing to some extent. On the other hand, the limitation of the present approach is that we have included only two lowest core states (two-core model), $p^{5}\left[{ }^{2} P_{3 / 2}\right]$ and $p^{5}\left[{ }^{2} P_{1 / 2}\right][14-16]$. Since it is possible nowadays to observe the fine-structure-resolved photoelec- 


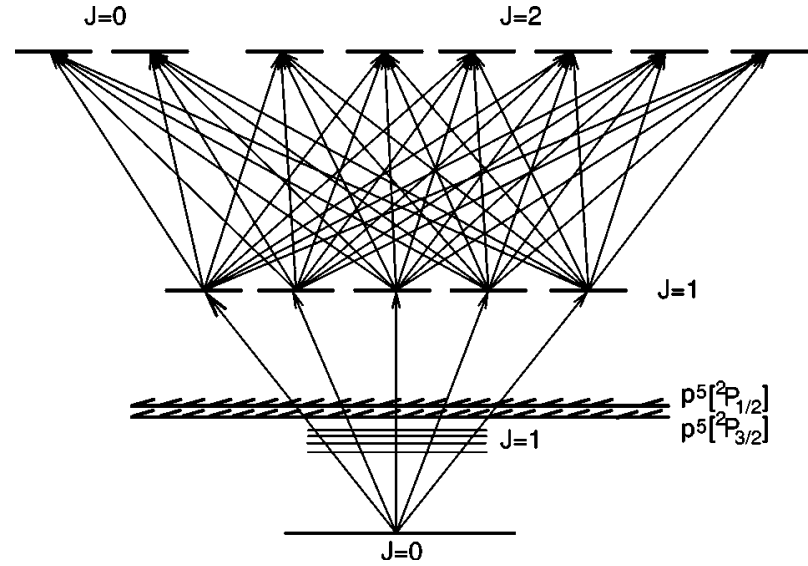

FIG. 1. MQDT description of the two-photon ATI process of Xe and Ar from the ground state.

tron spectra associated with the $p^{5}\left[{ }^{2} P_{3 / 2}\right]$ and $p^{5}\left[{ }^{2} P_{1 / 2}\right]$ core states [7], it is a reasonable and also practical approximation to neglect all other core states. In other words our two-core model implicitly assumes that the mixing of the configurations $p^{5} \epsilon l$ and $p^{4} n l \epsilon l^{\prime}$ are negligibly small.

The scheme we consider is depicted in Fig. 1 in terms of the MQDT picture. Rare-gas atoms such as Xe and Ar in the ground state $p^{6}(J=0)$ are exposed to xuv radiation with the photon energy of $25 \mathrm{eV}$. The single-photon absorption brings ground-state atoms to the continuum with $J=1$. Since we deal with the two-core model, there are five channels for the intermediate $J=1$ odd-parity continuum, i.e., $p^{5}\left[{ }^{2} P_{3 / 2}\right] \epsilon s_{1 / 2}$, $p^{5}\left[{ }^{2} P_{3 / 2}\right] \epsilon d_{3 / 2}, \quad p^{5}\left[{ }^{2} P_{3 / 2}\right] \epsilon d_{5 / 2}, \quad p^{5}\left[{ }^{2} P_{1 / 2}\right] \epsilon s_{1 / 2}, \quad$ and $p^{5}\left[{ }^{2} P_{1 / 2}\right] \epsilon d_{3 / 2}$. By absorbing an additional photon, the final continuum can be either $J=0$ or $J=2$. There are two channels for the $J=0$ even-parity continuum, i.e., $p^{5}\left[{ }^{2} P_{1 / 2}\right] \epsilon p_{1 / 2}$ and $p^{5}\left[{ }^{2} P_{3 / 2}\right] \epsilon p_{3 / 2}$, and six channels for the $J=2$ even-parity continuum, i.e., $p^{5}\left[{ }^{2} P_{3 / 2}\right] \epsilon p_{1 / 2}, \quad p^{5}\left[{ }^{2} P_{3 / 2}\right] \epsilon p_{3 / 2}, \quad p^{5}\left[{ }^{2} P_{3 / 2}\right] \epsilon f_{5 / 2}$, $p^{5}\left[{ }^{2} P_{3 / 2}\right] \epsilon f_{7 / 2}, p^{5}\left[{ }^{2} P_{1 / 2}\right] \epsilon p_{3 / 2}$, and $p^{5}\left[{ }^{2} P_{1 / 2}\right] \epsilon f_{5 / 2}$. Note that $\epsilon$ denotes energy of the photoelectron. In terms of the MQDT description, all transitions depicted by arrows in Fig. 1 are possible.

Briefly, the total MQDT wave function, $\Psi$, can be written as a linear combination of the wave functions for channel $\alpha$, $\Psi_{\alpha}[13]$, i.e.,

$$
\begin{aligned}
\Psi & =\sum_{\alpha} A_{\alpha} \Psi_{\alpha} \\
& =\sum_{i} \chi_{i}\left[f \sum_{\alpha} U_{i \alpha} \cos \pi \mu_{\alpha}-g \sum_{\alpha} U_{i \alpha} \sin \pi \mu_{\alpha}\right] A_{\alpha} \\
& =\sum_{\alpha} \sum_{i} \chi_{i}\left[f U_{i \alpha} \cos \pi \mu_{\alpha}-g U_{i \alpha} \sin \pi \mu_{\alpha}\right],
\end{aligned}
$$

where $\chi_{i}$ is a core state wave function and $A_{\alpha}$ is a suitable coefficient determined by the boundary conditions at $r \rightarrow \infty$. $U_{i \alpha}$ is a unitary transformation matrix that connects collision channels $i$ (in $j j$ coupling) and close-coupling channels $\alpha$ (nearly $L S$ coupling). $f$ and $g$ are the regular and irregular Coulomb functions. $\mu_{\alpha}$ is a quantum defect for channel $\alpha$. Needless to say, hydrogenic phase shifts are introduced for each channel. As a result of taking a sum over different channels, MQDT wave functions are different from the hydrogenic wave functions.

In order to compute the two-photon ATI transition amplitudes, we need the following quantities: (i) ground $(J=0)$-bound $(J=1)$ and (ii) ground $(J=0)$-free $(J=1)$ dipole moments for the first photoabsorption, and (iii) bound ( $J$ $=1)$-free $(J=0$ or 2$)$ and (iv) free $(J=1)$-free $(J=0$ or 2$)$ dipole moments for the second photoabsorption. Since MQDT does not generate the ground-state wave function, empirical dipole moments fitted to the experimental bound spectra have been used for (i). Similarly, for ground-free dipole moments (ii), experimental single-photon ionization cross section is in the literature as a function of photon energy [17]. However, more detailed information, namely the dipole moments for each channel, is not experimentally available. We have neither experimental information nor a simple way to analyze how the dipole moments for each channel behave in the energy region far above the ionization threshold. Therefore we have decided to fix their ratios and made the scaling as a function of photon energy so that the calculated cross sections agree with the experimental data. As for (iii), excited bound states and continuum wave functions are generated from the MQDT parameters and the dipole moments are calculated in the length gauge. Note that we have included the linear energy dependence of the MQDT parameters to describe the excited bound states. In contrast, the energy dependence of the MQDT parameters for the continuum states has been fixed to the values for the $p^{5}\left[{ }^{2} P_{1 / 2}\right]$ threshold, since, as in (ii), we have neither experimental information nor a simple way to analyze how the MQDT parameters behave in the energy region far above the ionization threshold. As for (iv), it is well known that the dipole moment does not converge if the length gauge is used, because both initial and the final continuum states extend to infinity. One way to circumvent this convergence problem for free-free transition is to employ the acceleration gauge. However, the acceleration gauge weights the contribution of the wave function for small $r$ which may not be accurate. Therefore we have made the combined use of the length and acceleration gauge $[18,19]$.

For the single-channel case, or for hydrogen, we calculate free-free dipole moments in the length gauge for small $r$ and in the acceleration gauge for large $r$, and combine them with appropriate surface terms. Namely, for the free-free transition from channel $i$ to channel $j$, the dipole moment in the length gauge, $D_{i, j}^{(L)}$ integrated over $0<r<\infty$, is decomposed into the quantities integrated over $0<r<R$ (inner region, typically $R=20-50$ a.u.) and $R<r<\infty$ (outer region), where the latter can be rewritten as a sum of the dipole moments evaluated in the acceleration gauge $D_{i, j}^{(A)}$ and the surface terms $S_{i, j}^{(V)}$ and $S_{i, j}^{(L)}$ :

$$
\begin{aligned}
D_{i, j}^{(L)} & =D_{i, j}^{(L)}(0, R)+D_{i, j}^{(L)}(R, \infty) \\
& =D_{i, j}^{(L)}(0, R)+\frac{D_{i, j}^{(A)}(R, \infty)}{4\left(E_{i}-E_{j}\right)^{2}}-\frac{S_{i, j}^{(V)}}{4\left(E_{i}-E_{j}\right)^{2}}-\frac{S_{i, j}^{(L)}}{2\left(E_{i}-E_{j}\right)},
\end{aligned}
$$

where 


$$
\begin{gathered}
D_{i, j}^{(A)}(R, \infty)=K_{i, j} \int_{r=R}^{\infty} d r F_{i}(r) \frac{4}{r^{2}} F_{j}(r), \\
S_{i, j}^{(V)}=K_{i, j}\left(2 F_{i}^{\prime}(R) F_{n}^{\prime}(R)-2 F_{i}(R) F_{j}^{\prime \prime}(R)+\frac{1}{R^{2}}\left[l_{i}\left(l_{i}+1\right)-l_{j}\left(l_{j}\right.\right.\right. \\
\left.+1)]\left\{R\left[F_{i}(R) F_{j}^{\prime}(R)-F_{i}^{\prime}(R) F_{j}(R)\right]-F_{i}(R) F_{j}(R)\right\}\right), \\
S_{i, j}^{(L)}=K_{i, j}\left\{F_{i}(R) F_{j}(R)+R\left[F_{i}(R) F_{j}^{\prime}(R)-F_{i}^{\prime}(R) F_{j}(R)\right]\right\},
\end{gathered}
$$

with $K_{i, j}$ being an angular coefficient for transition from $i$ to $j$, and $l_{i}$ the orbital angular momentum of the excited electron for channel $i . R$ is the inner box radius. $F_{i}(r)$ and $E_{i}$ are the radial wave function and the energy for channel $i . F_{i}^{\prime}(r)$ and $F_{i}^{\prime \prime}(r)$ are the first and second derivatives of function $F_{i}(r)$. As a check, we have ensured that our computer subroutine for free-free transition gives correct numbers for hydrogen. In terms of the convergence we have found that the choice of the inner box size $R=20$ a.u. is computationally economical, since it turned out that a very small mesh size is not required for the reasonable convergence. As for the outer region, Eq. (3), a very good convergence is easily obtained when the upper limit of $\infty$ is replaced by 200 a.u.. Having checked the convergence, we move on to the check of bound-free and free-free dipole moments for hydrogen by comparing our numbers with those obtained with the Greenfunction technique. Very good agreement (within 1\% difference) is obtained.

Now for the multichannel case, the MQDT wave function described above is expressed more explicitly as [15]

$$
\begin{aligned}
& \left|f_{m_{s}, m_{J_{c}}, J}(\hat{k}, r)\right\rangle=\sum_{l, m_{l}} i^{l} e^{-i \delta_{l}} Y_{l m_{l}}^{*}(\hat{k}) \sum_{j, J, \alpha} e^{-i \pi \mu_{\alpha}}\left(\alpha \mid J_{c} j J M_{J}\right) \\
& \times\left(J_{c} j J M_{J} \mid J_{c} m_{J_{c}} j m_{j}\right)\left(J_{c} m_{J_{c}} j m_{j} \mid l m_{l} s m_{s}\right)|\alpha\rangle,
\end{aligned}
$$

where $|\alpha\rangle=\Psi_{\alpha}$, and $\delta_{l}$ is the Coulomb phase shift and $\hat{k}$ $=(\theta, \varphi)$ characterizes the direction of the photoelectron with respect to the laser polarization axis for linear polarization. By implementing Eqs. (2)-(5) into Eq. (6), we can calculate free-free dipole moments for the multichannel case. The twophoton bound-bound-free transition amplitude for the multichannel case, $M_{b b f}^{(2)}$, can be calculated using the formula given by

$$
M_{b b f}^{(2)}=\sum_{k_{2}} \sum_{m} \frac{\left\langle f_{2}, k_{2}|D| m\right\rangle\langle m|D| g\rangle}{E_{g}+\omega-E_{m}},
$$

where $m$ is the intermediate bound states and $\left|f_{2}, k_{2}\right\rangle$ represents the final continuum of the channel denoted by $k_{2}$. All matrix elements are evaluated in the length gauge and $\omega$ is a photon energy in atomic units. Similarly, the two-photon bound-free-free transition amplitude for the multichannel case, $M_{b f f}^{(2)}$, can be calculated using the formula given by

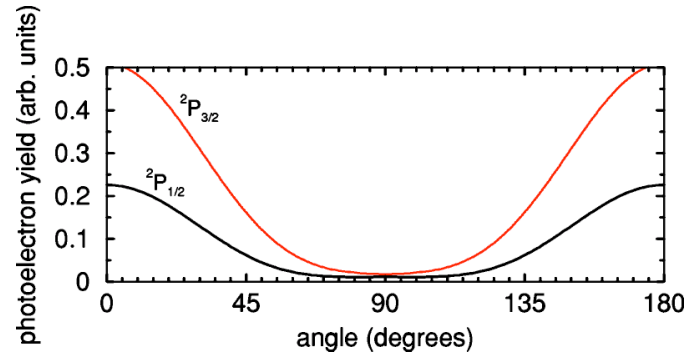

FIG. 2. Photoelectron angular distribution of the two-photon ATI of Xe by $25-\mathrm{eV}$ photons.

$$
\begin{aligned}
M_{b f f}^{(2)}= & \lim _{\epsilon \rightarrow 0+} \frac{1}{\omega^{4}} \sum_{k_{2}} \sum_{m_{1}, k_{1}} \frac{\left\langle f_{2}, k_{2}|A| m_{1}, k_{1}\right\rangle\left\langle m_{1}, k_{1}|A| g\right\rangle}{E_{g}+\omega-E_{m_{1}}} \\
= & \frac{1}{\omega^{4}}\left[P \sum_{m_{1}, k_{1}} \frac{\left\langle f, k_{2}|A| m_{1}, k_{1}\right\rangle\left\langle m_{1}, k_{1}|A| g\right\rangle}{E_{g}+\omega-E_{m_{1}}}\right. \\
& \left.-i \pi\left\langle f_{2}, k_{2}|A| m_{0}, k_{1}\right\rangle\left\langle m_{0}, k_{1}|A| g\right\rangle\right],
\end{aligned}
$$

where $m_{1}$ represents the intermediate continuum, and $m_{0}$ is the continuum at the pole with energy $E_{m_{0}}=E_{g}+\omega$. Note that all matrix elements are evaluated in the acceleration gauge. The total two-photon ATI transition amplitude is a sum of both bound-bound-free and bound-free-free transition amplitudes, i.e., $M^{(2)}=M_{b b f}^{(2)}+M_{b f f}^{(2)}$. In reality, it turned out that the summation for the intermediate continuum has to be taken up to $\sim 65 \mathrm{eV}$ from the ground state for the reasonable convergence of $M_{b f f}^{(2)}$ with $25-\mathrm{eV}$ photons, and $M_{b b f}^{(2)}$ is much smaller than $M_{b f f}^{(2)}$. Finally the two-photon ATI cross section we have obtained is $1.0 \times 10^{-51} \mathrm{~cm}^{4} \mathrm{~s}$ for Xe by $25-\mathrm{eV}$ photons. Similar calculations have been performed for $\mathrm{Ar}$, and we obtain $2.0 \times 10^{-51} \mathrm{~cm}^{4} \mathrm{~s}$. In Figs. 2 and 3 we show the angular distribution of two-photon ATI photoelectrons for Xe and Ar. One may wonder why angular distributions are so different for Xe and Ar. From Eqs. (6)-(8), however, this is not surprising, since the contribution of each channel to the twophoton ATI cross section is different for Xe and Ar. Most importantly, the values of quantum defect in Eq. (6), $\mu_{\alpha}$, are different for each channel of both atoms. The branching ratio, defined as the ratio of photoelectron yield leaving the $p^{5}\left[{ }^{2} P_{1 / 2}\right]$ core to that of the $p^{5}\left[{ }^{2} P_{3 / 2}\right]$ core, can be calculated from Figs. 2 and 3, and we obtain 0.42 for $\mathrm{Xe}$ and 0.38 for

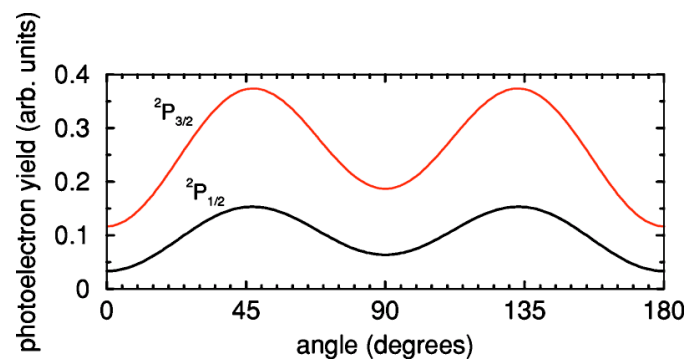

FIG. 3. Photoelectron angular distribution of the two-photon ATI of Ar by $25-\mathrm{eV}$ photons. 
Ar with $25-\mathrm{eV}$ photons, assuming a detection angle of \pm 12 degrees along the polarization axis. Compared with the experimental results reported in Ref. [7], the cross sections agree reasonablly well for both $\mathrm{Xe}$ and Ar while the branching ratios reveal some discrepancy. Nevertheless, taking into account the approximations we had to introduce to tackle the complexity of the problem, the overall results are reasonably good.

In summary we have calculated the two-photon ATI cross sections and photoelectron angular distributions of $\mathrm{Xe}$ and Ar within the extended framework of multichannel quantum defect theory (MQDT). The use of the extended MQDT enables us to distinguish photoelectron spectra associated with two ionic core states, $p^{5}\left[{ }^{2} P_{3 / 2}\right]$ and $p^{5}\left[{ }^{2} P_{1 / 2}\right]$. Free-free di- pole moments needed for the two-photon summation have been calculated with the combined use of the length gauge for small $r$ and the acceleration gauge for large $r$. The calculated two-photon ATI cross sections by $25-\mathrm{eV}$ photons are $1.0 \times 10^{-51} \mathrm{~cm}^{4} \mathrm{~s}$ and $2.0 \times 10^{-51} \mathrm{~cm}^{4} \mathrm{~s}$ for $\mathrm{Xe}$ and $\mathrm{Ar}$, respectively.

T.N. acknowledges useful discussions with L. A. A. Nikolopoulos regarding the combined use of the length and acceleration gauge. T.N. also acknowledges the hospitality by the members of Professor Watanabe's group at ISSP where part of this work was carried out. This work was supported by a Grant-in-Aid for Scientific Research from the Ministry of Education and Science of Japan.
[1] P. Agostini, F. Fabre, G. Mainfray, G. Petite, and N. Rachman, Phys. Rev. Lett. 42, 1127 (1979).

[2] K.C. Kulander, Phys. Rev. A 362726 (1987).

[3] H.G. Muller, Phys. Rev. A 60, 1341 (1999).

[4] X. Tang, H. Rudolph, and P. Lambropoulos, Phys. Rev. A 44, R6994 (1991).

[5] Y. Kobayashi, T. Sekikawa, Y. Nabekawa, and S. Watanabe, Opt. Lett. 23, 64 (1998).

[6] E. Takahashi, Y. Nabekawa, T. Otsuka, M. Obara, and K. Midorikawa, Phys. Rev. A 66, 021802(R) (2002).

[7] N. Miyamoto, M. Kamei, D. Yoshitomi, T. Kanai, T. Sekikawa, T. Nakajima, and S. Watanabe, Phys. Rev. Lett. 93, 083903 (2004).

[8] J. Andruszkow et al., Phys. Rev. Lett. 85, 3825 (2000).

[9] V. Ayvazyan et al., Phys. Rev. Lett. 88, 104802 (2002).
[10] V. Ayvazyan et al., Eur. Phys. J. D 20, 149 (2002).

[11] W. Nicklich, H. Kumpfmuller, H. Walther, X. Tang, Huale Xu, and P. Lambropoulos, Phys. Rev. Lett. 69, 3455 (1992).

[12] D.J. Kennedy and S. T. Manson, Phys. Rev. A 5, 227 (1972).

[13] M.J. Seaton, Proc. Phys. Soc. London 88, 801 (1966); Rep. Prog. Phys. 46, 167 (1983).

[14] O. Robaux and M. Aymar, Comput. Phys. Commun. 25, 223 (1982).

[15] A. L'Huillier, X. Tang, and P. Lambropoulos, Phys. Rev. A 39, 1112 (1989).

[16] T. Nakajima and P. Lambropoulos, Europhys. Lett. 57, 25 (2002).

[17] J.A. Samson and R.B. Cairns, Phys. Rev. 173, 80 (1968).

[18] M.J. Seaton, J. Phys. B 14, 3827 (1981).

[19] M.J. Seaton, J. Phys. B 19, 2601 (1986). 